\title{
EXPORTACIONES Y DESARROLLO DE LA PEQUEÑA EMPRESA NACIONAL
}

\author{
EXPORTS AND DEVELOPMENT OF NATIONAL SMALL BUSINESS
}

\author{
Julio Alberto Hennings Otoya* \\ Docente Auxiliar de la Facultad de Ciencias Contables- UNMSM
}

[Recepción: abril de 2011/ Conformidad: Mayo de 2011]

"No es valiente el que no tiene miedo, sino el que sabe conquistarlo" Nelson Mandela

\section{RESUMEN}

Ante las dificultades que muchas veces presenta el mercado peruano, dado su limitado tamaño para el desarrollo de la emergente pequeña empresa industrial, esta podría aprovechar las posibilidades que brinda el mercado ampliado de la Comunidad Andina -mayormente sin trabas arancelarias- para las empresas peruanas, planificando sus estrategias de desarrollo empresarial ${ }^{1}$ incursionando en los mercados vecinos, donde la similitud con los consumidores locales y la cercanía física, facilitaría conseguir resultados comerciales favorables.

En esta perspectiva, se analiza el impacto que pueden tener las exportaciones en su crecimiento de ventas, y como nuevo proveedor internacional, el desarrollo imprescindible de ventajas competitivas que le permitan ingresar a un primer mercado extranjero; asimismo, se revisan los cambios administrativos, productivos etc. congruentes con la nueva condición de empresa exportadora, y como estos repotenciaran la nueva organización.

Del trabajo, centrado en la pequeña empresa, se vierten conclusiones sobre las posibilidades que se le presentan a estas unidades con la exportación, a fin de aprovechar su poten-

\begin{abstract}
Given the difficulties that often presents the Peruvian market, given its limited size for the development of the emerging small industrial company, this could exploit the possibilities offered by the enlarged market of the Andean Community and mostly non tariff barriers for firms Peruvian planning their strategies for business development inroads into neighboring markets, where the similarity with local consumers and the physical proximity would facilitate achieving favorable business results.

In this perspective, we analyze the potential impact on export sales growth, and as new international supplier, essential to the development of competitive advantages that allow you to enter a foreign market first, also reviews the administrative changes, production etc. consistent with the new exporter status, and how these repower the new organization.

Work, focusing on small business, poured conclusions about the possibilities that are presented to the export units, in order to exploit their potential to pursue development and have an expanded international market.
\end{abstract}

\footnotetext{
Doctor en Ciencias Económicas - UFV, Docente de la Facultad de Ciencias Contables - UNMSM.

E- mail: jhennings@speedy.com.pe

1 Larousse 1995, Diccionario (vincula el término desarrollo a la mejora y al crecimiento), página 202
} 
cial para buscar el desarrollo contando con un extendido mercado internacional.

Palabras claves: Exportaciones, mypes, pymes, mercado CAN, ventajas competitivas.

\section{INTRODUCCION}

Una de las instituciones pioneras en llamar la atención respecto al tema de las posibilidades de las pequeñas empresas, y la necesidad de dotarlas de instrumentos de promoción para hacerlas competitivas, considerando el entorno macroeconómico de apertura por los años 90', fue el COPEI de la Sociedad Nacional de Industrias (SIN), en la que en documento titulado "Visión Estratégica para el Desarrollo y la Promoción de la Pequeña Empresa, señalaba que la pequeña empresa tenía escasa participación en el esfuerzo exportador peruano y que "debería empezarse por los instrumentos que signifiquen abrir nuevos mercados y oportunidades para lograr la necesaria competitividad en el mercado globalizado ${ }^{2 "}$.

Sobre este tema de las empresas exportadoras, es importante señalar que ya en el
Key words: Export MYPES, PYMES, CAN market, competitive advantages.

año 2004, según datos de la SUNAT, solo el $0.26 \%$ de las Mype's (micro y pequeña empresa) en el Perú tenían una orientación exportadora:

Han pasado algunos años desde los primeros esfuerzos en descubrir el potencial de nuestras pequeñas empresas en las exportaciones nacionales, sin embargo, el escenario actual nos muestra que aun su participación es escasa (alrededor del 10\% del valor exportado). Esto, considerando su elevado número -6,656 empresas en el 2007 representando el 97\% del total de exportadores (Promperu)-, no hace sino reafirmar el carácter emprendedor de muchos empresarios pymes, aunque su competitividad empresarial no sea aun eficaz en la arena internacional. Un ejemplo para esta afirmación se da con la información oficial de aduanas, cuando vemos que el promedio exportador en el mismo año 2007,

Empresas y exportadores en el Perú (2004)

\begin{tabular}{|l|r|r|r|}
\hline $\begin{array}{l}\text { RANGO DE } \\
\text { VENTAS }\end{array}$ & $\begin{array}{r}\text { NUMERO DE } \\
\text { EMPRESAS }\end{array}$ & $\begin{array}{r}\text { NUMERO DE EMP. } \\
\text { EXPORTADORAS }\end{array}$ & $\begin{array}{r}\text { ORIENTACION } \\
\text { EXPORTADORA } \\
\text { B/A }\end{array}$ \\
\hline MICRO & 622,209 & 918 & 0.14 \\
\hline PEQUEÑA & 25,938 & 756 & 2.91 \\
\hline SUBTOTAL MYPE & $\mathbf{6 4 8 , 1 4 7}$ & $\mathbf{1 , 6 7 4}$ & $\mathbf{0 . 2 6}$ \\
\hline MED+GRAN & 10,899 & 1,383 & 12.69 \\
\hline TOTAL & 659,046 & & 3,057 \\
\hline
\end{tabular}

Fuente: SUNAT / Elaboración Propia

2 COPEI - SNI 1997, Visión Estratégica Para el Desarrollo y Promoción de la Pequeña Empresa, Pag.55-57 
de la mypes era apenas de 113,000 dólares anuales, monto visiblemente insuficiente para generar ahorros para su desarrollo.

Lo anterior nos hace pensar que la pequeña empresa aún no está aprovechando los beneficios del mercado global (mayor demanda, diversificación de plazas, etc.) ni tampoco el acceso preferencial que tenemos a naciones como EE.UU. (TLC) o las de la Unión Europea (SGP Plus). Si bien la participación de las pymes peruanas en las exportaciones se encuentra dentro del promedio en América (incluyendo los EE.UU), está bastante distanciada de la que alcanzan las pymes europeas y las asiáticas, las cuales deberían de ser nuestras referentes dado el espíritu emprendedor en nuestro país -según The Global Entrepreneurship Monitor (GEM) ubican al Perú entre los países con más emprendedores del mundo (4 de cada 10 peruanos desarrolla actividades de emprendimiento). Además de contar en el país con la fortaleza de una variada gama de recursos minerales, agrícolas, pesqueros, turísticos etc., que nos sitúan entre los primeros lugares del mundo.

Sobre las motivaciones que estarían limitando el crecimiento exportador de las

\section{Participación de la Pymes en las exporta- ciones totales de cada país}

$\begin{array}{lc}\text { País } & \text { Porcentaje } \\ \text { Brasil } & 9 \% \\ \text { Argentina } & 10 \% \\ \text { EE.UU. } & 11 \% \\ \text { Francia } & 26 \% \\ \text { Corea del Sur } & 40 \% \\ \text { Italia } & 53 \% \\ \text { Taiwán } & 56 \%\end{array}$

Fuente: SEPyME y CEP - http://www.ordonez-bianco.com/herramientas/indicador

pymes nacionales, hemos encontrado un estudio realizado en el 2008 por el Consorcio de Investigación Económica y Social (CIES), donde se muestra la percepción de factores que habrían impedido la exportación de confecciones -rubro muy importante- de numerosas pymes. El principal motivo lo atribuyen a la falta de apoyo gubernamental, entendido como la ausencia de una política de Estado que favorezca la exportación de las

\section{Factores que han impedido la exportación de confecciones de las pymes (según los que no exportan)}

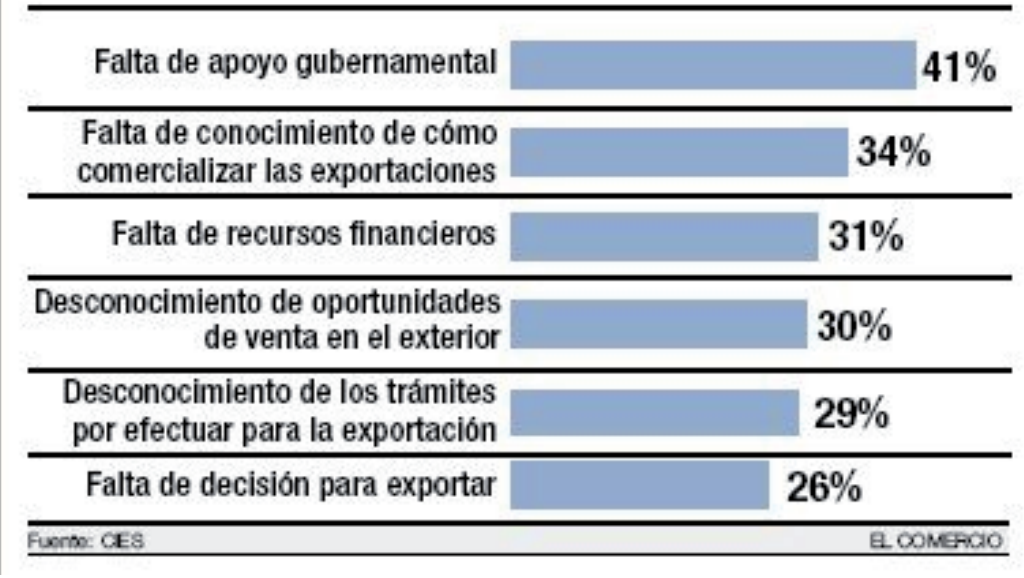


pymes. Sin embargo, si analizamos las demás razones vemos que de los seis factores respondidos, cuatro corresponden a la falta de conocimiento y un quinto factor, que podría estar influido por el mismo anterior se refiere a la falta de decisión.

Esto guarda relación con la apreciación personal, que a la pequeña empresa productiva de nuestro país, les está faltando conocimiento y experiencia exportadora para aprovechar los beneficios de la mundialización. Con la explotación de nuevos mercados para sus productos, facilitaría su crecimiento y la generación de excedentes que permita que ingresen al circulo virtuoso de: + ventas, + productividad $\mathrm{y}+$ ventas.

\section{ANTECEDENTES TEORICOS}

Sobre la necesidad de dotar de fortaleza competitiva internacional a la industria, debemos citar a Peter Drucker, cuando señala que como consecuencia de la existencia de una sola economía y un mercado, "todo negocio tiene que ser globalmente competitivo, aunque fabrique o venda solo dentro de un mercado local o regional"3. Esto tiene mayor fundamento, en cuanto al mercado peruano, así como en el mercado andino que se pretende incursionar, puesto que concurren bienes de diversos países.

Sobre la decisión de la pequeña empresa productiva nacional, para escoger un país limítrofe con características e idiosincrasia de mercado similar al propio, para iniciar su expansión de ventas, encontramos al Profesor Paliwoda de la Universidad de Calgary ${ }^{4}$ cuando menciona que "buscar la atención de los consumidores en países que comparten el mismo perfil en términos de ingreso, prestigio y estilo de vida es más seguro que llegar a un mercado extranjero con la espada desenfundada para llegar a personas desconocidas, que es lo que se hace con un enfoque de ventas no orientado...”.

Es decir, al fijar el objetivo comercial de la pyme peruana de expandirse al mercado andino considerando a clientes potenciales similares a los que atiende en el Perú, se está minimizando el riesgo que puede traer el incursionar inicialmente en otro mercado alternativo, cuyas características y preferencias podrían ser muy diferentes con el propio mercado local original, por lo que se considera que la estrategia de segmentación estandarizada sería una arma de mercadotecnia importante, proporcionando la oportunidad de un ataque bien preparado en un mercado objetivo y una defensa adecuada frente a los competidores potenciales que operan en dicho mercado.

Porter al referir las ventajas competitivas en el comercio internacional, considera que la productividad es el factor que refleja el desarrollo y crecimiento económico de un país. Manifiesta que la productividad refleja: "la capacidad de la industria para innovar y mejorar", todo lo cual garantizará "el objetivo principal de la nación, producir un alto y creciente nivel de vida para sus ciudadanos"s.

El crecimiento sostenido de la productividad requiere que una empresa se perfeccione continuamente. Esta productividad se alcanzará mediante la elevación de la calidad de los productos, la adición de características deseables, la mejora de la tecnología del producto o superación de la eficiencia de la producción. La productividad debe verse como

3 Peter F. Drucker - 2002, “La Gerencia en la Sociedad Futura” / Pag. 12

4 Stanley J. Paliwoda - 1996, "La Esencia de la Mercadotecnia Internacional” / Pag. 41

5 Porter Michael 1990, “¿Dónde radican las ventajas competitivas de las naciones?. Harvard-Deusto Business Review, 4a Trimestre, página 16. 
un todo en la cadena de valor y en darle al cliente el mejor producto que este puede obtener y en las mejores condiciones.

Esta productividad se verá incrementada por el comercio internacional en donde la empresa en cuestión no solo competirá con sus pares a nivel nacional sino que competirá contra empresas que poseen otros recursos, otras ventajas comparativas que sabrán desarrollar diferentes ventajas competitivas respecto del resto de los competidores.

Estas conclusiones van centrando la atención sobre que estrategia debe aplicar la Pyme peruana que desea comercializar con el extranjero para alcanzar el éxito competitivo. Al respecto, la teoría nos dice que las empresas deben poseer una ventaja competitiva en forma de costo inferior, o bien de productos diferenciados que obtengan precios superiores.

\section{Investigaciones sobre las pymes exporta- doras}

El desarrollo empresarial de la pequeña empresa industrial facilitado por las exportaciones, ha sido investigado desde hace varios años en el Perú por el Profesor Alan Fairlie. En su publicación "Competitividad e Integración: Las Pymes Industriales Exportadoras del Perú “", señala que el principal destino de las exportaciones de dichas unidades es el mercado andino y que los principales efectos en las empresas investigadas habían sido:

- Aumento en la escala de producción.

- Mayor especialización en las líneas de producción de la empresa.

- Reducción de costos unitarios por un aumento del tamaño del mercado.

- Se adoptó nueva tecnología productiva.

- Se renovaron equipos.
- Se dio un aumento del número de unidades producidas por hora trabajador.

En cuanto a los beneficios en su proceso operativo interno, condicionados por la estrategia competitiva que tuvieron que adoptar para las exportaciones, las pequeñas empresas:

- Capacitaron en mayor medida al personal.

- Reorganizaron el área administrativa.

- Implementaron o mejoraron el área de post venta.

- Mejoraron el sistema de comercialización y distribución.

Es decir, se consiguió una mejora importante en el capital humano, vía procesos de capacitación para poder acceder a mercados externos, y un manejo administrativo más técnico.

Los resultados de esta investigación, refuerzan la hipótesis que con las exportaciones iniciales al mercado andino (Ecuador, Colombia y Bolivia) las pymes peruanas pueden mejorar su posición competitiva, abriéndoles el camino para un crecimiento empresarial sostenido.

\section{EL PROBLEMA VINCULADO A SU DESARROLLO}

En el Perú, según el Ministerio de Producción el $98.6 \%$ de todo el tejido empresarial esta constituido por la micro y pequeña empresa - mypes, la cual contribuye con el $77 \%$ del empleo (incluyendo el autoempleo) y aportan el $42.1 \%$ del PBI del país.

Estas empresas abastecen en diferentes rubros (alimentos, confecciones, accesorios metálicos para industria del calzado etc.) al mercado peruano, aunque también una parte

6 Alan Fairlie Reinoso, noviembre 2003, Competitividad e Integración: Las Pymes Industriales Exportadoras del Perú - Pags, 74-75-76-93-95 
importante de este, es abastecido por la empresa corporativa y por las importaciones, con gran incidencia las de origen asiático, sobre todo en los sectores ligados a las confecciones de calzado y ropa. Adicionalmente, se sabe que existe cierto grado de informalidad en estos últimos, cuyos estimados indican que las ventas no registradas llegarían al 5.6\% de las adquisiciones reales, conformada por el $2.8 \%$ de importaciones de contrabando ${ }^{7}$, y el resto a fabricantes que no declaran sus ventas o lo hacen parcialmente, lo que abona en contra de la transparencia de la competencia.

Este tema de concurrencia en el limitado mercado peruano, de los mencionados productos de fabricación formal nacional, conjuntamente con los de producción extranjera y peor aún los informales, afecta las posibilidades de crecimiento empresarial de las Mype's peruanas. Por un lado, estas industrias locales se ven envueltas en una competencia difícil, donde cada cual oferta precios más bajos, -sin privilegiar, calidad o presentación-, obligándose a aceptar márgenes de utilidad muy estrechos, que restringen su posibilidad de ahorro para la inversión. Y por otro lado, un mercado compartido entre tantos ofertantes, implica reducidas probabilidades de incrementar ventas y por ende, dificultades para costos productivos de "escala”, más bajos.

Como alternativa a la restrictiva condición del mercado local, para el crecimiento de las Mype's se plantea exportar inicialmente al mercado andino, donde la cercanía física, puede facilitar el acceso logístico. Asimismo, existen facilidades para las empresas de la Comunidad Andina, como las preferencias acordadas (exoneraciones) a la importación de la mayor parte del universo arancelario ${ }^{8}$, lo que contribuiría a la tendencia ascendente de las exportaciones peruanas a ese bloque.

\section{FACTORES PROPUESTOS EN EL PLANTEAMIENTO}

Factores con impacto positivo para el desarrollo empresarial, impulsados por la exportación de las Mypes a los países de la CAN:

\section{Factores de Impacto en la Exportación}

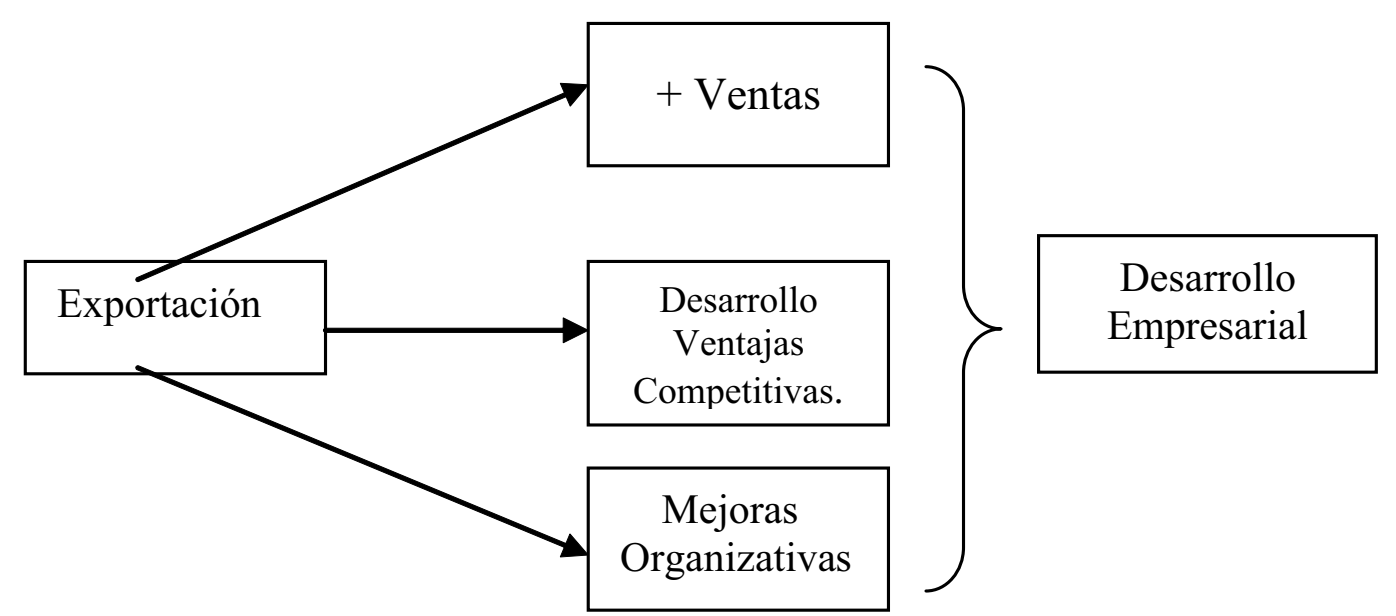

Fuente: Hennings, Julio 2006. Impacto de las exportaciones al Ecuador en el desarrollo de mype fabricante de ojalillos.

7 INS Gerencia de Estadística. 2001 Una Posible Aproximación al Nivel de Contrabando en el Perú

8 CAN-Per Dec. Ej. 692 y 1329, R.O. 166 y 296 de Oct-03-97, Oct-12-99 (Web: sice1.aduana.gov.ec) 


\section{DESARROLLO DE LOS FACTORES PROPUESTOS}

\section{Fortalezas que definirán las ventas}

En primera instancia, dada la situación actual del Perú, con un tipo de cambio estable, alta desocupación (equivalente a mano de obra barata y abundante), e insumos con precios internacionales, hace suponer que las mypes deberían centrarse en costos bajos. Bajo estas premisas el productor peruano estaría en inmejorables condiciones de ofertar en el mercado internacional algunos productos competitivos en costos, sin embargo, esto no determina que el mismo pueda mantener dicha ventaja en el tiempo.

Esto es fácil de predecir cuando vemos la evolución que tuvieron los precios a nivel internacional de los productos de mayor tecnología e incluso de tecnología intermedia, como las confecciones textiles o el calzado y como cambió la economía de los mismos cuando países como China, Brasil y la India ingresaron agresivamente en los mercados mundiales. Inclusive la realidad actual es que los países que no tienen los factores naturales $\mathrm{u}$ otro factor comparativo, a diferencia del Perú, tienen gobiernos que intervienen activamente en el desarrollo de sus economías sectoriales ayudándolos e igualándolos con incentivos y subsidios (ejemplos de esto son China y la Unión Europea).

La ventaja competitiva basadas en costos, es una ventaja efímera, ya que la empresa que hoy día se beneficia con el menor costo de mano de obra, se verá rápidamente desplazada por la que lo tenga mañana. Asimismo, la fuente más barata para el aprovisionamiento de un recurso natural puede cambiar de la noche a la mañana, en el caso de que una nueva tecnología permita la explotación de ese recurso en lugares que hasta entonces se hubieran considerado imposibles o antieconómicos. En sectores sensibles al costo de los factores, el liderazgo suele cambiar rápidamente.

Independientemente del comentario anterior, elegir basarse en la diferenciación no eliminará el incentivo (beneficio) a mediano y largo plazo de producir en el fabricante nacional, ya que este al haberse decidido por una estrategia distinta a la de centrarse en costos, verá como la demanda de sus productos centrados en una estrategia de diferenciación, perduraran en el tiempo, haciendo esta actividad sostenible en el largo plazo.

Este aspecto de la sostenibilidad empresarial en el horizonte de tiempo, tal vez tenga que ver con la desilusión del $20 \%$ de las pymes que abandonan el mercado al cuarto año de su primera exportación ${ }^{9}$, cuando notan que los beneficios son limitados y por tanto su futuro es incierto.

\section{Creación de Ventajas Competitivas}

Las empresas crean valor para sus compradores por medio de la realización de determinadas actividades. El valor definitivo que crea un productor se mide por el precio que los compradores están dispuestos a pagar por sus productos.

Un negocio es rentable si el precio de venta supera el costo colectivo de realización de todas las actividades requeridas. Las actividades llevadas a cabo por el productor pueden agruparse por categorías conformando lo que Porter denomina "la Cadena de Valor" ${ }^{10}$. Todas las actividades de la cadena de valor contribuyen a acrecentar el valor para el comprador. Las actividades pueden

9 http://www.pymex.pe/noticias/peru/2579-conociendo-a-las-pymes-exportadoras-

10 Porter Michael E. 1987, Ventaja Competitiva, Pág. 52 
dividirse, a grandes rasgos, en dos grupos: aquellas que se refieren a la producción, comercialización, entrega y servicio de posventa del producto, dentro de un plano cotidiano (actividades primarias) y aquellas que proporcionan recursos humanos, tecnología e insumos comprados, o funciones generales de infraestructura para apoyar las otras actividades.

La estrategia marca la forma en que una empresa realiza sus peculiares actividades y organiza toda su cadena de valores. Los enlaces se producen cuando la forma de llevar a cabo una actividad afecta los costos o la eficacia de otras actividades. Obviamente, los enlaces exigen también que las actividades estén coordinadas. Las entregas a tiempo reducen inventarios de seguridad muy importantes. Todo esto reduce costos de transacción, mejoramiento en la información y del control, necesarios para la seguridad en el lanzamiento. La hábil gestión de los enlaces puede ser una fuente decisiva de ventaja competitiva. Muchos enlaces no son evidentes y los competidores a veces tienen dificultades para percibirlos.

La unión de la cadena de valor de un productor con sus proveedores que aportan insumos, su canal de distribución y con el comprador final del producto conforma lo que se denomina "sistema de valor".
Obtener y mantener la ventaja competitiva depende de no solo comprender la cadena de valor de una empresa, sino como encaja una empresas en el sistema de valor general.

La ventaja competitiva es, cada vez más, una función de lo bien que un productor pueda gestionar todo este sistema. Un productor puede crear ventajas competitivas mediante la optimización o coordinación de estos enlaces con sus clientes en el exterior. Pero las oportunidades de conseguir economías mediante la coordinación con los proveedores y los canales van más allá de la logística y el tratamiento de los pedidos, y abarcan la investigación y el desarrollo de nuevos productos, el servicio posventa y muchas otras actividades.

La cadena de valor ofrece un medio de comprender las fuentes de ventajas en costos, ya que lo habitual es centrarse en los costos de producción, mientras que si vemos toda la cadena de valor veremos que existen muchos otros costos además de los costos de producción, más aun si extendemos el estudio de los costos al sistema de valor. Desde que un producto cualquiera se produce hasta que llega al consumidor, va sumando costos como el de procesamiento, transporte, embalaje, almacenamiento, controles de certificación $y$ otros que hacen que el producto final de comercialización resulte tener un costo muy

Sistema de Valor

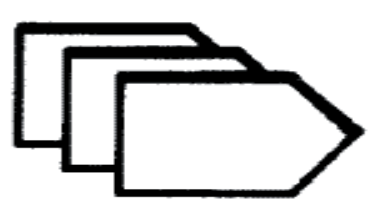

CADENAS DEL VALOR DE LOS PROVEEDORES

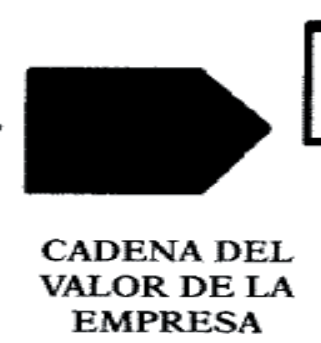

EMPRESA

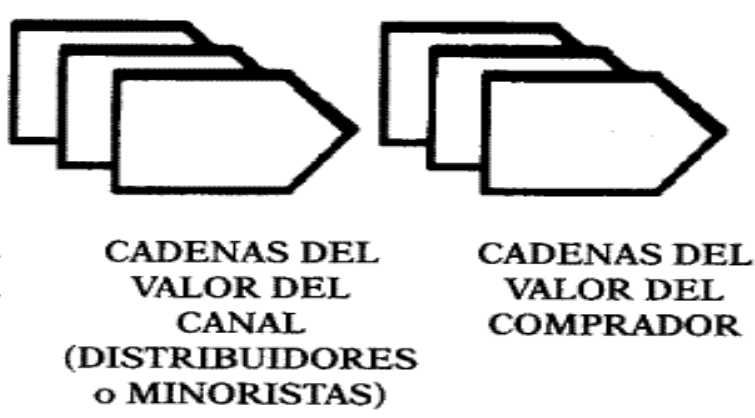

Fuente: M. Porter 1987, Ventaja Competitiva, Pág. 53 
superior al costo de producción. Es en este punto donde toma sentido decir que la productividad, está en la cadena de valor y sus sistemas de valor.

La cadena de valor también pone en evidencia las fuentes de diferenciación, como podría ser el canal de comercialización elegido o el tipo de envase seleccionado o bien el precio final al consumidor.

El análisis de todos los factores, de la cadena de valor y sus sistemas, de las fuerzas que afectan al mercado y de la formas diferentes que descubren los productores para ser los mejores en cada punto, son la forma en que estos crean sus ventajas competitivas. Nuestros pequeños productores por tanto, deben innovar en cada aspecto, investigando nuevas y mejores formas de competir y así mantener su competitividad en el tiempo. Ellos deben poner énfasis en las nuevas tecnologías, en las nuevas o cambiantes necesidades del comprador, en la aparición de nuevos segmentos u oportunidades comerciales, en los cambios en los costos o disponibilidades de los insumos, en los cambios en las disposiciones gubernamentales etc.

Ahora bien la sola obtención de una ventaja competitiva no garantiza la perpetuidad de la misma, sino que muy por el contrario estas cambian permanentemente, dejando al productor sin su ventaja competitiva. Entonces debemos decir que la sustentabilidad de la ventaja competitiva depende de tres condiciones:

1) La "fuente" específica de la ventaja: hay jerarquías de fuentes de ventajas competitivas en términos de sustentabilidad. Las ventajas de orden inferior, tales como los costos de mano de obra o de materias primas baratas, son fáciles de imitar. Las ventajas de orden superior, tales como la tecnología de proceso propia de la empresa, la diferenciación de productos basada en esfuerzos de marketing acumulados, y las relaciones con los clientes protegidas por los altos costos que para éstas representaría el cambio de proveedor, son más duraderas. Estas, para ser alcanzadas por la competencia requieren de técnicas y capacidades más avanzadas, tales como personal especializado y de elevada formación, capacidades técnicas interna $y$, frecuentemente, estrechas relaciones con los clientes líderes.

2) El "número de diferentes fuentes" de ventajas de que dispone una empresa: si una empresa cuenta con una sola fuente de

\section{La Cadena de Valor}

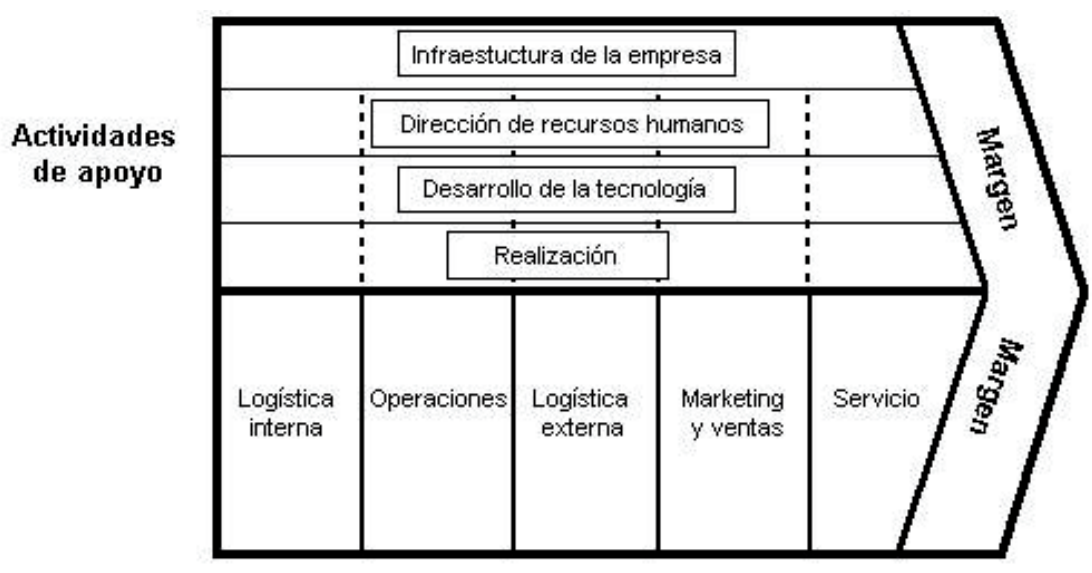

Actividades primarias

Fuente: M. Porter 1987 Ventaja Competitiva 
ventaja, los competidores se dedicarán a anular dicha fuente y al final la empresa perderá la totalidad de sus ventajas competitivas. Las empresas que permanecen por varios años como líderes en un merado, tienen diversas fuentes a lo largo de toda su cadena de valor.

3) La "mejora y el perfeccionamiento constante": esta es la más importante de las tres condiciones para mantener ventajas competitivas en el tiempo. Tarde o temprano cualquier ventaja puede verse replicada si un líder se duerme en los laureles. Con objeto de mantener sus ventajas, los productores deben ser blancos móviles y crear nuevas ventajas al menos tan de prisa como sus competidores puedan imitar las antiguas.

\section{Adaptación de la organización}

El nuevo esquema de actuación en un mercado ampliado (CAN), requerirá cambios en el sistema de gestión y de adaptación de las nuevas exportadoras pymes, introduciendo el proceso de aprendizaje valioso, que le posibilitaría contar con la experiencia necesaria para incursionar en otros mercados de exportación en el futuro.

\section{Implantación de Cambios y Nuevas Políticas}

Con el fin de cubrir las expectativas sobre los nuevos productos de exportación, la gerencia de las pequeñas exportadoras deben investigar los factores determinantes que incidan en su ventaja futura, incorporando por ejemplo en los bienes que ofrecen algunos cambios que satisfagan mejor a sus clientes potenciales; estos, servirán como guía para el desarrollo de ventajas frente a la competencia. Asimismo, requerirán establecer nuevas políticas internas que estén alineadas a los objetivos de optimizar la atención del nuevo mercado, como:

1. La atención de pedidos (entregas de producción) de exportación es prioritaria frente a los pedidos locales. Esto debido que el proceso logístico del comercio internacional requiere mayor tiempo.

2. Adaptar de inmediato la infraestructura administrativa y productiva de la empresa (insumos, maquinaria y personal) al nuevo nivel conjunto de operaciones para la atención (despacho) local e internacional, privilegiando la cobertura de servicio de atención frente a un probable incremento de costos. Por ejemplo,

\section{Diagrama de requisitos para la oferta exportable}

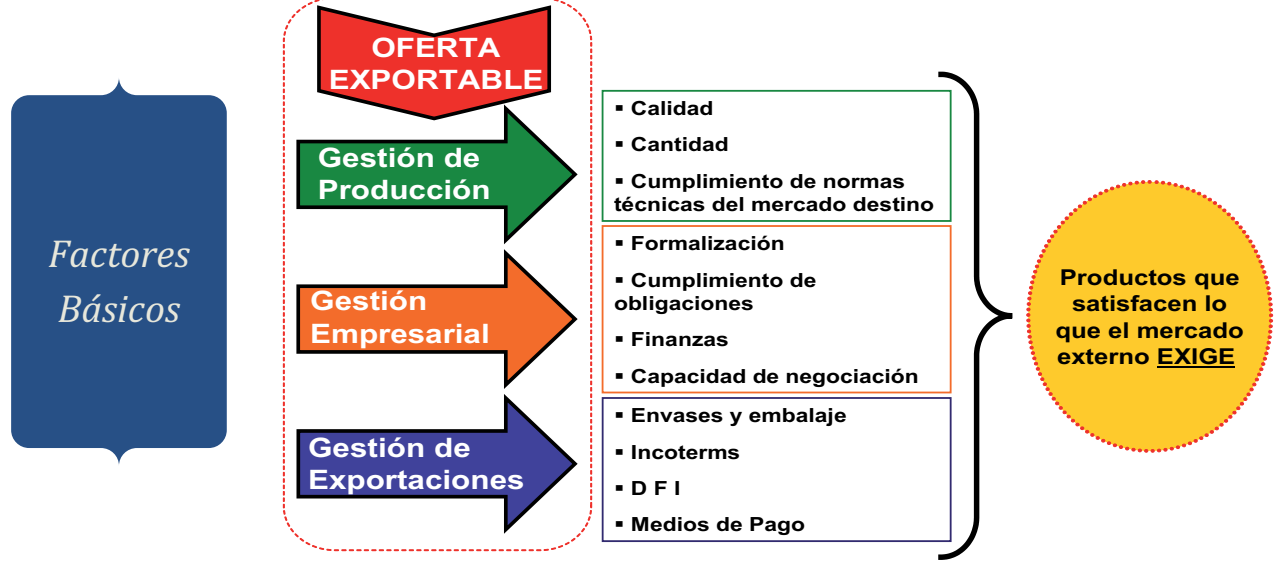

Fuente: Prompex / Elaboración Propia 
si se requiere compras adicionales -no negociadas inicialmente con precios formalizados-, $\mathrm{u}$ horas extras de personal se procederá a asumirlas.

3. Determinar simultáneamente las nuevas necesidades de personal en las áreas de línea y apoyo, para hacer los ajustes organizacionales que se requieran, considerando las nuevas funciones generadas por las exportaciones.

4. Establecer incentivos para los trabajadores de la empresa que colaboren eficazmente en al proceso exportador.

5. Diseñar y establecer reportes de control y monitoreo de "Clientes de Exportación", que acompañen a los pedidos atendidos, a fin que los mismos clientes participen en la evaluación de la calidad de los productos recibidos y el nivel de servicio de entrega (cantidad recepcionada, demora, empaque, documentos remitidos y sugerencias), que le permitan a la pyme exportadora identificar sus debilidades y buscar mejoras continuas para la creación de vínculos de fidelización con sus nuevos clientes.
6. Un aspecto importante que acerca al cliente potencial, mejora la comunicación e incrementa las posibilidades de ventas con menores costos es el que se relaciona con la tecnología informática, así por ejemplo tener un Portal (interactivo) Web, le permitirá a la empresa mostrar al mundo sus productos, características técnicas y aplicaciones en tiempo virtual, lo cual podría constituirse en una excelente presentación para iniciar una rentable relación de cliente-vendedor.

\section{CONCLUSIONES}

1. Las exportaciones de la pequeña empresa industrial en el mercado andino, puede producir resultados positivos reflejados principalmente en:

- Mayores ventas totales (por la contribución de las exportaciones).

- Desarrollo de ventajas competitivas, resaltando en:

a. Menores costos de sus productos (por economías de escala).

b. Mejoras de la oferta por nuevas exigencias competitivas.

\section{MODELO DE EJEMPLO}

\begin{tabular}{|c|c|c|c|c|c|c|c|}
\hline IGSA & & & & & & & \\
\hline \multicolumn{8}{|c|}{ REPORTE DE CLIENTES DE EXPORTACION } \\
\hline \multicolumn{8}{|l|}{ EMBARQUE $\mathrm{N}^{\circ}$} \\
\hline \multicolumn{8}{|c|}{ FECHA DE ШEGADA } \\
\hline \multicolumn{8}{|c|}{ EMPRESA CLIENTE } \\
\hline \multicolumn{8}{|c|}{ SOBRE SUPEDIDO RECEPCIONADO } \\
\hline \multirow[t]{2}{*}{ PRODUCTO (S) } & \multicolumn{2}{|c|}{ CANTIDADES } & \multicolumn{5}{|c|}{ CONFORMIDAD O OBSERVACIONES RESPECTO A: } \\
\hline & ORDENADAS & RECIBIDAS & CALIDAD & ENVASE & DOCUMENT & DEMORA & OTROS \\
\hline & & & & & & & \\
\hline & & & & & & & \\
\hline & & & & & & & \\
\hline & & & & & & & \\
\hline & & & & & & & \\
\hline \multicolumn{8}{|c|}{ ESTIMADO PARA SU PROXIMO PEDIDO: } \\
\hline & & & & & & & \\
\hline \multicolumn{8}{|c|}{ FECHA APROXIMADA DE REQUERIMIENTO } \\
\hline & & & & & & & \\
\hline \multicolumn{8}{|c|}{ CANTIDAD PLANIFICADA } \\
\hline \multirow{2}{*}{\multicolumn{8}{|c|}{ SUGERENCIAS PARA MEJORAR ATENCION }} \\
\hline & & & & & & & \\
\hline \multirow{2}{*}{\multicolumn{8}{|c|}{ r }} \\
\hline & & & & & & & \\
\hline \multicolumn{8}{|c|}{ 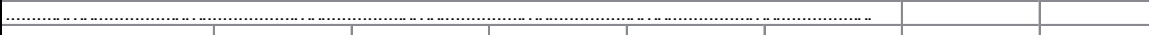 } \\
\hline & \multicolumn{3}{|c|}{ NOMBRE DE FUNCIONARIO (CLIEN TE) ... } & & \multicolumn{3}{|l|}{ CARGO.......... } \\
\hline
\end{tabular}


c. Mejoras en la comunicación y desarrollo de comercio virtual.

d. Eficiente atención de servicio al cliente

e. Incremento de capacidad productiva.

f. Incremento en la capacidad de respuesta al cambio.

- Mejor organización funcional, y elevación del nivel profesional de la organización.

2. Las mayores ventas, consecuentes de participar exitosamente en los mercados local e internacional y la reducción de los costos, le permitirán a la empresa, incrementar su rentabilidad.

3. La suma de factores benéficos generados por las exportaciones, podrán facilitar a la pequeña empresa nacional emprender el camino hacia un desarrollo empresarial sostenido.

4. El desarrollo de ventajas competitivas para participar en un mercado externo con competidores internacionales, contribuyen a mejorar la posición de la empresa en el mercado interno, generando el consiguiente incremento de clientes y ventas locales.

5. Las mejoras de la organización y la calificación conseguidas en el proceso de adaptación hacia una empresa exportadora, permiten realizar una gestión más eficiente de recursos, posibilitando rendimientos superiores en los procesos y actividades, facilitando con ello el logro de los objetivos empresariales.

6. Prepararse competitivamente para participar en un primer mercado externo obliga a superar muchas debilidades internas y a reforzar las fortalezas de la empresa, permitiendo además obtener una experiencia importante para seguir desarrollándose en el mercado global.

\section{REFERENCIAS BIBLIOGRAFICAS}

1. Arellano Cueva, Rolando. 2001, Comportamiento del Consumidor Enfoque América Latina, México, Editorial Mc Graw-Hill, 457 páginas.

2. Aduanas - INS Gerencia de Estadística. 2002, Una Posible Aproximación al Nivel de Contrabando en el Perú: 2001, Lima, 27 páginas.

3. CAN - Secretaría General. 1999, Como hacer Negocios en la Comunidad Andina: Guía de Comercio e Inversión, Lima, 187 páginas.

4. COPEI -SIN. 1998, Visión Estratégica Para el Desarrollo y Promoción de la Pequeña Empresa, Lima, 76 páginas.

5. Drucker, Peter F. 2002, La Gerencia en la Sociedad Futura, Bogotá, Grupo Editorial Norma, 290 páginas.

6. Fairlie Reinoso, Alan. 2003, Competitividad e Integración: Las Pymes Industriales Exportadoras en el Perú, Lima, Pontificia Universidad Católica del Perú, 186 páginas.

7. Hammer, Michael \& Champy, James. 1994, Reingeniería, Bogotá, Grupo Editorial Norma, 226 páginas.

8. Hennings, Julio. 2006. Impacto de las Exportaciones al Ecuador en el Desarrollo de Mype Fabricante de Ojalillos. Tesis 2006. UNFV.

9. Paliwoda Stanley J. 1996, la Esencia de la Mercadotecnia Internacional, Prentice Hall Hispanoamericana S.A., 164 páginas.

10.Porter, Michael E. 1987, Ventaja Competitiva: Creación y Sostenimiento de un Desempeño Superior, México, Compañía Editorial Continental S.A., 550 páginas.

11. Prompex - Olegario Llamazares 2004, Como Negociar con Éxito en 50 Países, Perú, Edición Especial Global Marketing Strategies, 230 páginas. 